\title{
Alteración de rocas graníticas utilizadas en edificación
}

\section{Alteration of granite stone used in building construction}

\author{
R. M. ${ }^{\text {a Esbert Alemany }}{ }^{(*)}$
}

Recepción/Received: 26-III-07

Aceptación/Accepted: 19-VII-07

RESUMEN

En el artículo se sintetizan los diversos aspectos que inciden en la alteración de las rocas graníticas, cuando estas se utilizan en los edificios, ya sea en forma de sillares o de losetas de revestimiento. Se hace especial hincapié en las anisotropías, fundamentalmente de carácter fisural que suelen presentar los granitos. Éstas suelen estar relacionadas con las condiciones estructurales del yacimiento del cual proceden. Dichas anisotropías condicionan el desarrollo de determinadas formas de alteración que con el paso del tiempo se aprecian, sobre todo en los sillares. También se recuerda que la petrografía, y en especial la composición química de los minerales que componen las rocas graníticas, condicionan su alteración, y que dentro del término comercial granito se suelen incluir otras rocas más alcalinas denominadas genéricamente granitoides y más alterables que el granito sensu-stricto. En cuanto a las propiedades físicas de estas rocas, cuya cuantificación es necesaria para evaluar su durabilidad, se muestra que algunas de ellas pueden presentar valores diferentes y relacionados con su anisotropía. Finalmente se revisan las formas, causas y mecanismos implicados en el deterioro de estas litologías cuando se utilizan como elementos constructivos.

Palabras clave: rocas graníticas, alteración, anisotropía, petrografía y propiedades físicas.
SUMMARY

The article contains a synthesis of the different factors involved in the alteration of granite ashlars or cladding used in building construction. Emphasis is placed on the -primarily fissure-like- anisotropies often present in granite, which are usually related to the structural conditions of the quarry where it is mined. Such anisotropies condition the development of certain types of alterations that appear over time, particularly in ashlars. Stone petrography, particularly as regards the chemical composition of the minerals comprising granite, also govern its alteration. Moreover, the commercial term granite often includes other more alkaline rocks generically known as granitoids, whose alterability is higher than in granite per se. The values of some of the physical properties of these rocks, which must be quantified to evaluate their durability, are shown to differ in keeping with their anisotropy. Finally, the forms, causes and mechanisms involved in granite decay are reviewed in the context of its use in building construction.

Keywords: granite, alteration, anisotropy, petrography, physical properties.

(*) Universidad de Oviedo (Asturias, España).

Persona de contacto/Corresponding author: resbert@geol.uniovi.es 


\section{INTRODUCCIÓN}

Las rocas graníticas se han utilizado en edificación desde tiempos remotos, especialmente en las zonas donde había afloramientos graníticos próximos al lugar donde se edificaba. En la actualidad y en forma de losetas de revestimiento los granitos se utilizan en todo el mundo existiendo vías de comercialización entre todos los continentes.

Estas rocas son el resultado de procesos geológicos generados en la corteza terrestre. Sus propiedades físicas y químicas dependen de dicha génesis, que se manifiesta en el tipo y porcentajes de minerales que entran en su composición, en sus características texturales y en las condiciones de emplazamiento en la corteza terrestre. Desde el punto de vista geológico se definen, como "aquellos materiales geológicos de origen ígneo, plutónico, holocristalinos, granulares, que poseen composición química ácida (rica en $\mathrm{SiO}_{2}$ ), con cuarzo, feldespatos (alcalinos y plagioclasa) y que pueden contener minerales máficos (ricos en hierro)". Esta definición sólo comprende algunos de los granitos ornamentales, los que podrían denominarse granitos sensu stricto y un conjunto de las denominadas rocas afines o granitoides, tales como granodioritas, cuarzo dioritas, monzonitas, sienitas. Sin embargo desde el punto de vista industrial el significado es más amplio: la normativa europea (EN 12670:2001, apartado 2.1.156) (1) define comercialmente al granito ornamental como una piedra natural compacta y que admite el pulido, utilizada en decoración y construcción, que fundamentalmente está formada por minerales con una dureza entre 5 y 7 en la escala de Mohs, correspondiente al feldespato, micas y cuarzo, respectivamente. Otras rocas plutónicas, rocas volcánicas con estructura porfirítica y rocas metamórficas con composición mineralógica similar a los granitos y granitoides como el gneis e incluso algunas calizas cristalinas entran, según la norma, dentro de esta denominación. Las que, siendo cristalinas, son oscuras, ultrabásicas, con abundantes minerales ferromagnesianos y con elevada densidad, se denominan comercialmente granitos negros.

Cuando las rocas graníticas se encuentran en la corteza, son duras, resistentes, muy compactas y nada porosas, pero cuando afloran a la superficie, "por el desmantelamiento de las rocas suprayacentes", se produce una descompresión desarrollándose, en los cuerpos graníticos, fisuras y aumentando así la porosidad del material. Estas fisuras van a actuar de dos maneras: por un lado, reduciendo la resistencia mecánica de la roca; y por el otro, facilitando el acceso a su interior de los agentes de alteración (agua, sales, ácidos húmicos, contaminantes, etc.). Dichas fisuras se encuentran en toda la masa granítica, incluso a escala de roca matriz ${ }^{1}$, donde suelen tener aperturas muy pequeñas (microfisuras).

Como se citó al principio, las rocas graníticas son materiales ampliamente utilizados en edificación, especialmente en España, país con numerosos afloramientos de estas litologías. Se ha utilizado a lo largo de la historia, así el Acueducto de Segovia, las catedrales de Santiago de Compostela, Orense, etc., el Monasterio de San Lorenzo de El Escorial, las fachadas del Museo del Prado, la Plaza Mayor de Madrid, el Palacio del Mayorazgo en Cáceres, etc., se han construido con sillares de este tipo de piedra. Más recientemente, con el avance de la tecnología, el granito se comercializa en forma de losetas y se usa también, entre otras aplicaciones, para el revestimiento de exteriores. Son ejemplos de esta utilización el Museo de Arte Contemporáneo de Santiago de Compostela, el edificio de La Presidencia del Gobierno Insular Canario en Las Palmas, La Casa de Las Ciencias en La Coruña, el Ayuntamiento de Tokio, etc.

\section{ASPECTOS QUE INCIDEN EN LA ALTERACIÓN DE LAS ROCAS GRANÍTICAS DE LOS EDIFICIOS}

Aunque el granito es una de las rocas más durables de las utilizadas en edificación, con el paso del tiempo suele mostrar diferentes lesiones, cuya implantación e intensidad varían de un edificio a otro, pero que pueden relacionar siempre con las características de cada tipo de granito, con la climatología y demás características ambientales -presencia de contaminantes-, por ejemplo, del entorno donde se ubica y también con las características específicas del edificio del que forman parte, tales como, la geometría de la superficie de las diferentes unidades arquitectónicas, la orientación del paramento, etc. (2) y (3).

En este trabajo se estudian las características intrínsecas del granito que influyen en su deterioro, las cuales se citan y describen a lo largo del apartado.

\subsection{Anisotropías del granito}

A primera vista puede parecer que esta roca está completamente exenta de discontinuidades. Sin embargo, se conoce que casi todos los granitos tienen características direccionales. Así, en las grandes masas graníticas existe una alineación de fisuras macroscópicas "exfoliación macroscópica" llamada por los americanos (rift) y unos "planos de crucero" (grain), perpendiculares a los anteriores. Por lo general, son, en la mayoría de cuerpos graníticos, vertical una y horizontal la otra. El plano perpendicular a ambos se llama, desde el punto de vista geológico, "plano de exfoliación difícil" (hard) (4).

1. Roca matriz: volumen de roca representativa del conjunto y libre de discontinuidades de macizo. 
Dale que realizó un intenso estudio sobre estas características en los granitos de Nueva Inglaterra se encontró con que las estructuras rift y grain, a escala de roca matriz, consisten en menudas grietas que atraviesan los granos de cuarzo, se extienden también en los feldespatos produciendo una red en dos direcciones perpendiculares. Las líneas de rift son, por lo general, paralelas a las láminas de mica. Dentro del rift y grain se forman minerales secundarios tales como sericita, limonita, etc.

"Rift" y "grain" se producen aparentemente en granitos que cristalizan bajo tensiones corticales y son, por tanto, características que la roca ha tenido desde su formación. Algunas de estas anisotropías texturales condicionan la alteración y explotación de los macizos graníticos, tal como puede apreciarse en las Figuras 1 y 2.

También en las masas graníticas se encuentran diaclasas, que son grietas producidas en el efecto de las tensiones regionales. Suelen producirse en más de una dirección, a veces son frecuentes dos direcciones perpendiculares; a veces las direcciones son completamente irregulares. Estas diaclasas condicionan la explotación en cantera pero no tienen ninguna significación en la roca matriz, porque precisamente la explotación evita estas discontinuidades para obtener los bloques dimensionados (Figura 3).

Un aspecto muy importante a considerar en las diaclasas es su distanciamiento (densidad). Si las diferentes familias de diaclasas están espaciadas de 3 a $9 \mathrm{~m}$, los bloques pueden ser removidos fácilmente; se obtienen bloques rectangulares y la explotación puede realizarse sistemáticamente. Una elevada densidad de diaclasas hacen a la roca despreciable para la explotación en bloques.

Las anisotropías estructurales del granito no se obvian completamente cuando éste se corta en volúmenes predeterminados para construir o para revestir. Las direcciones de anisotropía preferentes, antes mencionadas, vienen marcadas, como ya se dijo, por la alineación de fisuras o de algunos minerales constituyentes y están total o parcialmente incluidas en la unidad constructiva.

Así pues, a escala de roca matriz, es decir, de roca "aparentemente" libre de discontinuidades de macizo, estas anisotropías suelen estar presentes y es necesario detectarlas antes de cualquier estudio petrofísico o alterológi$\mathrm{co}$, porque marcan direcciones con comportamientos diferenciales. Las anisotropías fisurales suelen determinarse

Tabla 1

Valores de la velocidad de propagación de ondas medidos en el granito de Axeitos

(La Coruña), según las tres direcciones del espacio

\begin{tabular}{|c|c|c|c|}
\hline \multicolumn{3}{|c|}{ vp (m/s) } \\
\hline Tipo Roca & Dirección $\mathbf{( X )}$ & Dirección (Y) & Dirección (Z) \\
\hline Granito (muestras seca) & $2.250 \pm 230$ & $2.600 \pm 220$ & $3.000 \pm 220$ \\
\hline Granito (muestras saturadas) & $3.990 \pm 380$ & $4.700 \pm 740$ & $5.100 \pm 280$ \\
\hline
\end{tabular}

X: dirección perpendicular a la microfisuración.

Z: dirección paralela a la microfisuración.

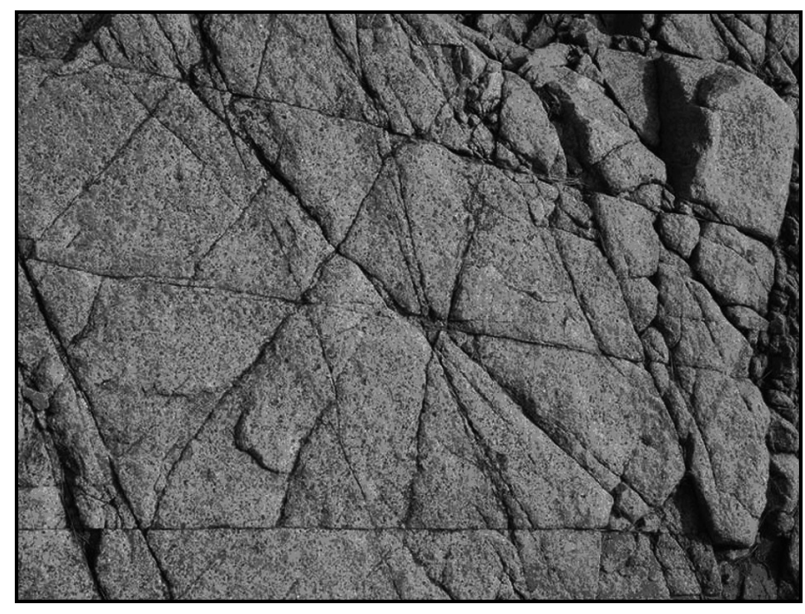

Figura 1. Direcciones de "rift" (tendencia vertical) y "grain" (tendencia horizontal), en un talud granítico. Se aprecian también diaclasas generadas por la tectónica regional. Cadena litoral catalana.

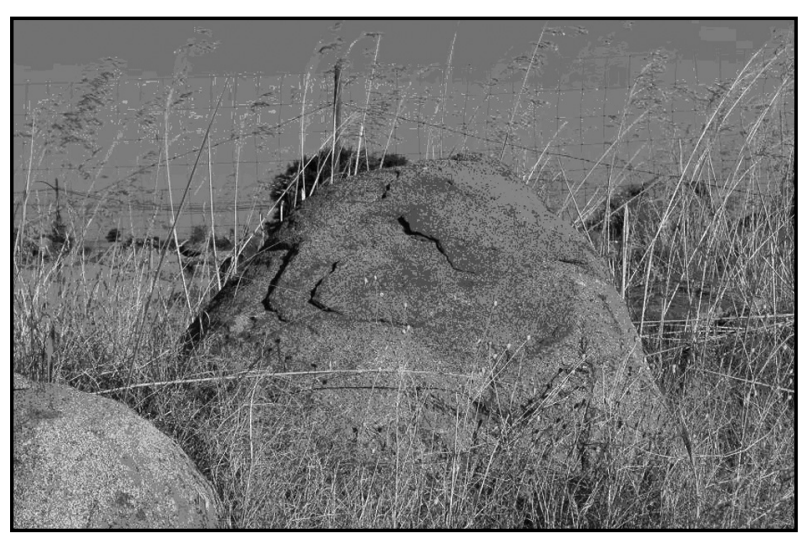

Figura 2. Disyunción bolar del granito generada por la presencia de direcciones de fisuración en la masa del granito, que generan estos bolos. Sierra de Guadarrama (Segovia). 
aplicando ultrasonidos, concretamente midiendo la velocidad de propagación de las ondas longitudinales en las tres direcciones del espacio. La Figura 4 muestra la posición probeta/transductor en la medida de $v_{p}$ en ensayos realizados en el laboratorio. En la tabla Tabla 1 se recogen los valores de $v_{p}$ medidos en las tres direcciones principales de los bloques de granito de Axeitos (La Coruña), en muestras secas y saturadas en agua.

Como puede constatarse, los valores difieren según la dirección considerada. La velocidad de propagación de ondas elásticas es siempre mayor en la dirección paralela a la alineación fisural ( $\mathrm{Z}$ ) que en las dirección perpendicular (X). Aunque la proporción se mantiene en el sentido que se acaba de indicar, la velocidad de propagación de las ondas longitudinales $\left(v_{p}\right)$ suele ser más alta en muestras saturadas en agua que en muestras secas (5), por lo que al realizar los ensayos deberá conocerse el contenido en agua de los bloques o de las probetas.

\subsection{Petrografía y textura del granito}

El granito es una roca plutónica, es decir, procede del enfriamiento de un magma intruido en la corteza terrestre, por lo que tiene carácter cristalino, sus minerales son por lo general idiomorfos, y están en contacto unos con otros sin una fase aglomerante de unión, como suele ser el caso de areniscas y calizas.

Es una roca polifásica, compuesta por tres minerales esenciales: cuarzo, feldespato y mica. El feldespato está representado por la ortosa y/o microclina (silicatos alumínico-potásicos) y por las plagioclasas (silicatos sódicocálcicos). Las micas son biotita y moscovita (silicatos con aluminio, hierro, magnesio y potasio) (Figuras 5, 6 y 7). Estos minerales tienen unas propiedades y comportamientos muy diferentes; mientras el cuarzo presenta una alta resistencia a la alteración química, una estructura

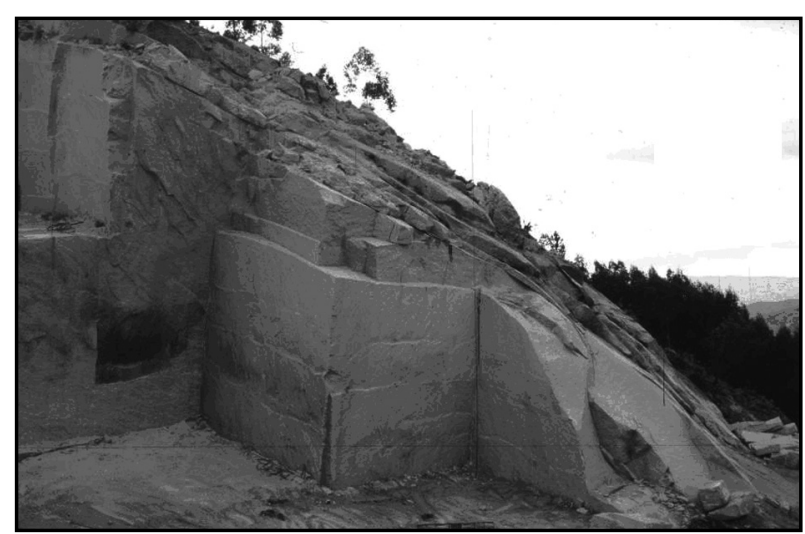

Figura 3. Aspecto de una cantera de granito. Porriño (Pontevedra). En la parte superior se observa la lajación generada por la descompresión del cuerpo granítico. La zona de explotación está libre de discontinuidades macroscópicas. estable y una baja solubilidad, los feldespatos son mucho más alterables; el feldespato potásico se hidroliza y pasa a caolinita; la plagioclasa es químicamente poco estable y también se hidroliza fácilmente en superficie dando minerales de la arcilla tales como: vermiculita + montmorillonita y si el proceso continúa, caolinita + gibbsita. La mica (biotita y/o moscovita) también se altera. La biotita se hidroliza pasando a clorita y lixiviando hierro; la moscovita presenta fenómenos de hidratación, oxidación, lixivación y cuando la alteración es muy acusada, se hidroliza e hidrata generando minerales secundarios como la vermiculita, caolinita y montmorillonita.

La alteración física del cuarzo y los feldespatos se manifiesta por la presencia de microfisuras de distinta apertura y recorrido. En algunos feldespatos, plagioclasas fundamentalmente, se observan hidromicas tipo sericita que al alterarse, dejan pequeños poros que por lo general se ubican en el centro de los cristales. En cuanto a las micas, los espacios entre las láminas de filosilicato pueden albergar agua que si pasa a hielo, ejerce esfuerzos sobre el mineral que se acaba desmoronando (Figura 8).

El tamaño de grano incide también en el deterioro. Los granitos y rocas afines suelen presentar tamaños de grano fino, medio, grueso y muchas veces los hay porfídicos (Figuras 9,10 y 11). A medida que aumenta el tamaño de grano, las rocas graníticas se hacen más susceptibles al deterioro porque algunos de los minerales más abundantes -feldespatos y micas - presentan mayores superficies de exposición y, por lo tanto, de ataque frente a los agentes de alteración.

Los granitos presentan en su textura espacios vacíos, mayoritariamente de tipo fisural, en los que se puede depositar o circular agua. El número de fisuras comunicadas no es muy alto, los valores de porosidad abierta en estas rocas suelen oscilar entre el $0,3 \%$ y el $5 \%$. Estos

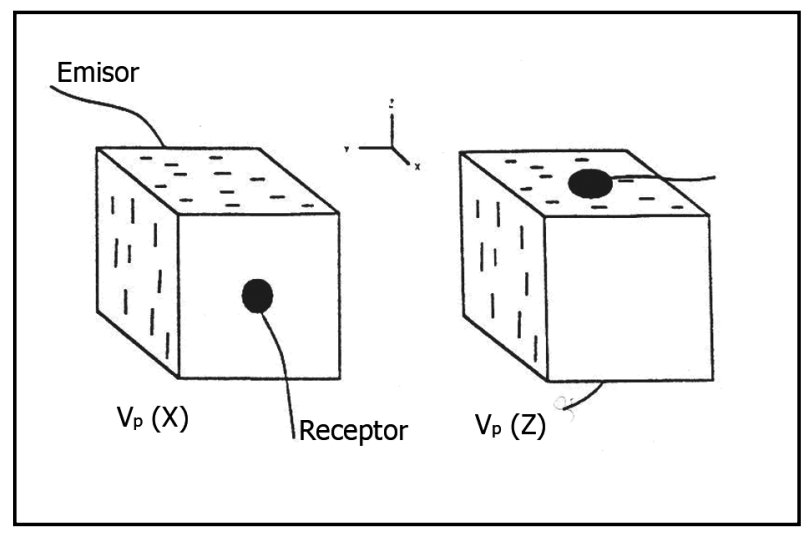

Figura 4. Esquema con la posición probeta/transductor en la medida de vp en un granito con microfisuras orientadas. 

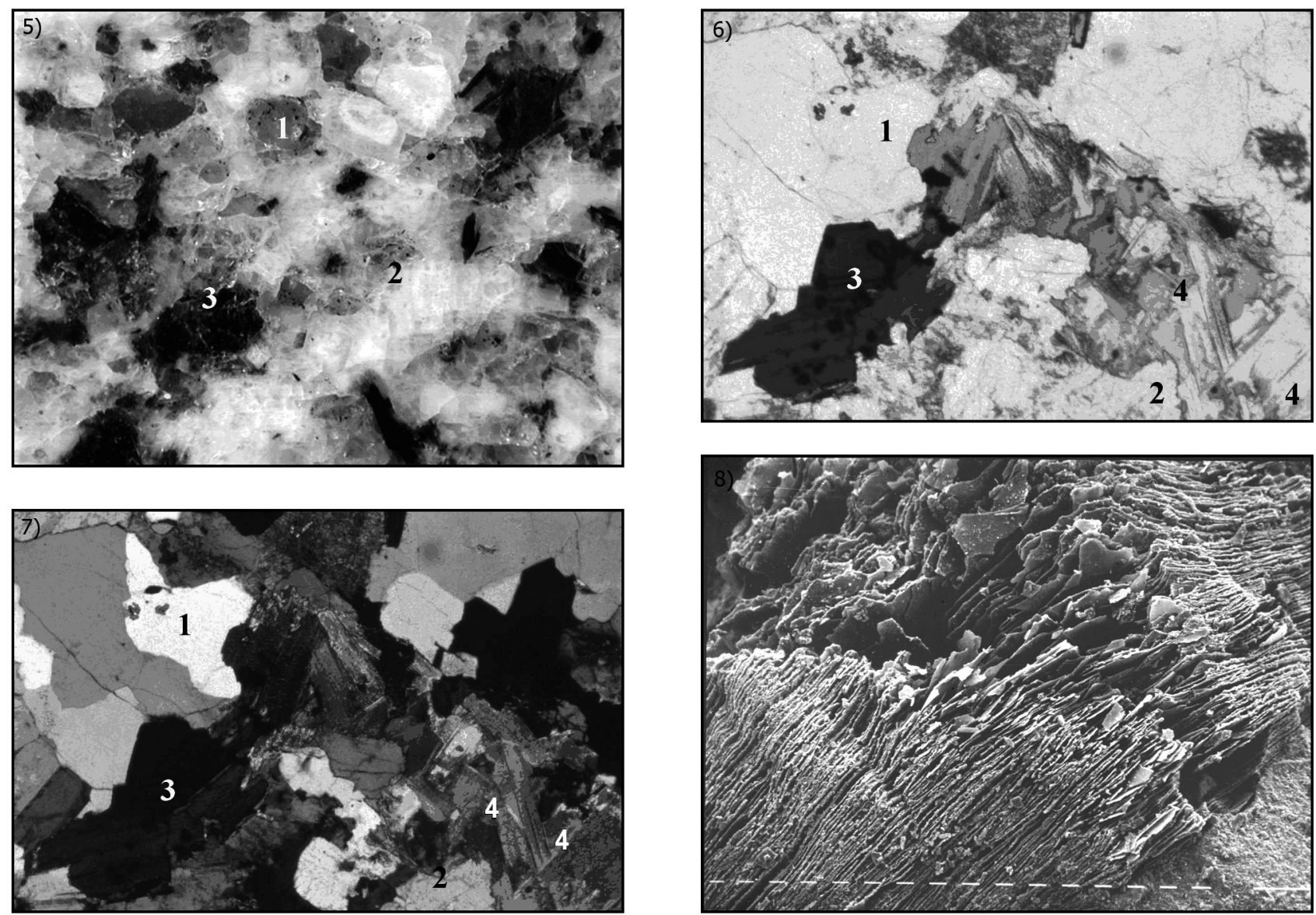

Figuras 5 a 8. 1) Figura 5: Aspecto macroscópico de los componentes de un granito: (1) cuarzo, (2) feldespato

(ortosa y microclina) y (3) mica (biotitas); Figuras 6 y 7: Aspecto al microscopio óptico de polarización (POL) de la mineralogía y textura de un granito de dos micas. Se diferencian los cristales de cuarzo (1), feldespato (2) y micas -biotita (3) y moscovita (4)-

y la textura granuda holocristalina. Figura 6: luz polarizada sin analizador y Figura 7: luz polarizada con analizador (x32).

La Figura 8 muestra el aspecto de un cristal de biotita al microscopio electrónico de barrido (SEM). Se aprecian las distintas láminas del filosilicato y los espacios vacíos entre ellas. $(1$ micromarcador $=10 \mu \mathrm{m}$.)

valores frente a los que presentan otras rocas empleadas en edificación como calizas y areniscas, que es frecuente que tengan valores de porosidad próximos al 30-35\%, son bajos, por lo que se puede afirmar que los granitos presentan porosidades bajas y, por lo general, sus espacios abiertos presentan mala comunicación. La microscopía electrónica de barrido, la óptica de fluorescencia y el láser confocal ayudan a visualizar y cuantificar estos espacios vacíos (Figuras 12 y 13).

\subsection{Composición química del granito y rocas afines}

La composición química del granito, ligada a su composición mineral, influye en su deterioro tal como se menciona en el apartado anterior. Además, los granitos utilizados en edificación, tanto en épocas históricas como recientes, en muchos casos no son granitos "sensu stricto", sino que son rocas cristalinas afines, tales como: granodioritas, cuarzodioritas, dioritas, etc., es decir, lo que en Petrología se denominan granitoides.
En ellos el porcentaje de cuarzo y feldespatos alumínico-potásicos decrece a medida que aumenta el carácter básico de los granitoides en relación con el granito, aumentando el de feldespatos sódico-cálcicos (plagioclasas). El porcentaje de los feldespatos más cálcicos (más alterables) crece frente a los más sódicos (Figura 14). Así, pues, según la roca utilizada sea un granito o un granitoide, la susceptibilidad al deterioro será menor o mayor.

Los granitoides pueden presentar, además de biotita y/o moscovita, otros minerales máficos como anfíboles, especialmente hornblenda.

A continuación (Tabla 2) se recoge la composición química, expresada en porcentajes de óxidos mayores, de diferentes tipos de rocas plutónicas: granitos, granitoides (granodioritas, sienitas y dioritas) y de los denominados, en el ámbito de las rocas ornamentales, granitos negros (gabros) (6). 


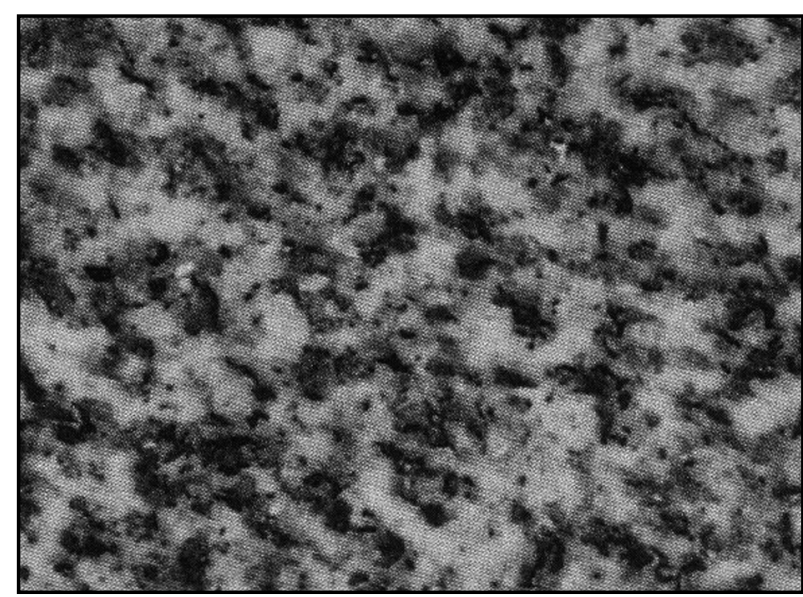

Figura 9. Aspecto macroscópico de un granito de grano fino (tamaño medio de grano inferior a $1 \mathrm{~mm}$ ).

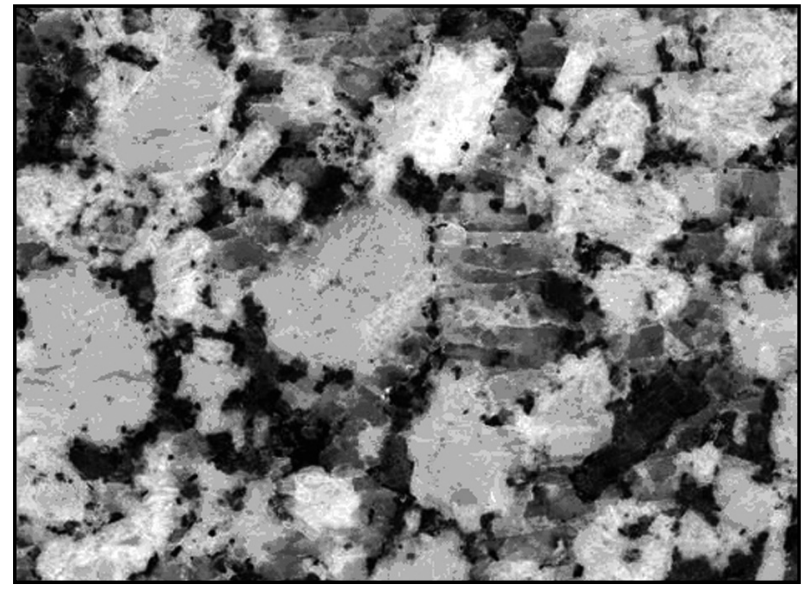

Figura 10. Aspecto macroscópico de un granito de grano medio-grueso (tamaño medio de grano entre 5 y $30 \mathrm{~mm}$ ).

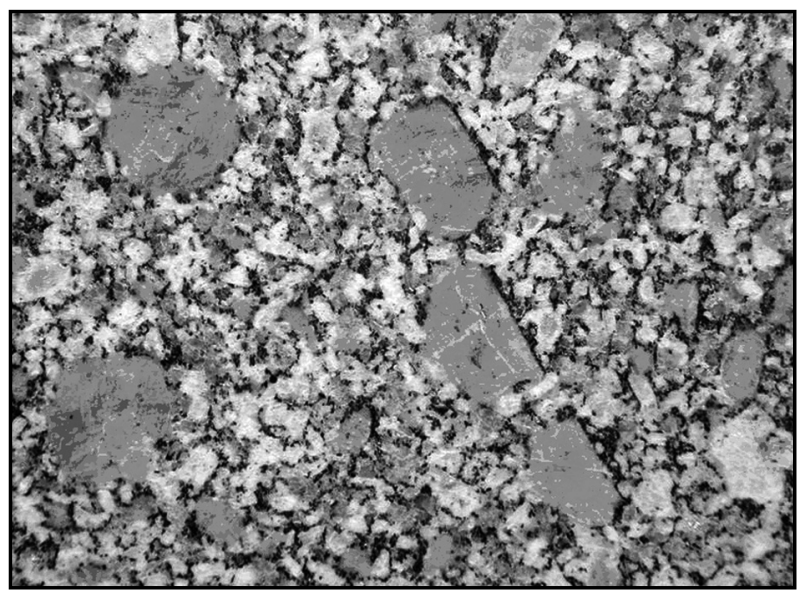

Figura 11. Aspecto macroscópico de un granito porfídico, respectivamente del filosilicato y los espacios vacíos entre ellas. $(1$ micromarcador $=10 \mu \mathrm{m}$.)
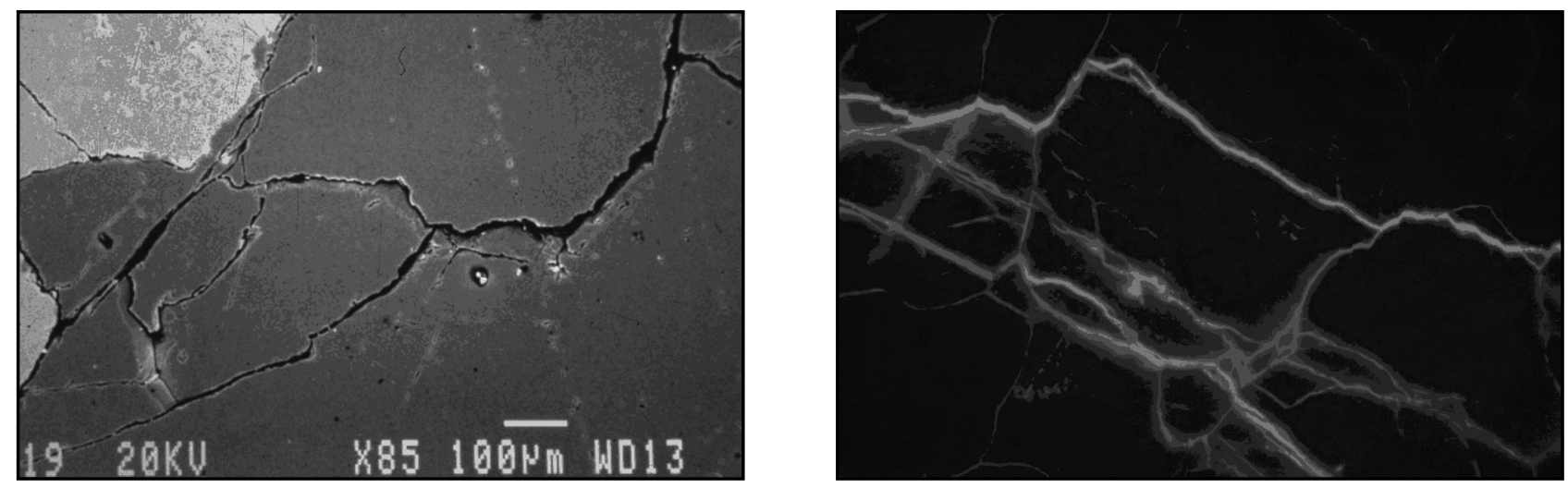

Figuras 12 y 13. Dos imágenes de los espacios vacíos (fisuras), característicos de las rocas graníticas. 12) microfisuras observadas al microscopio electrónico de barrido (SEM) y 13) microfisuras observadas con microscopía óptica de polarización (POL) y luz fluorescente.

El porcentaje de cuarzo $\left(\mathrm{SiO}_{2}\right)$ disminuye del granito al gabro, a la vez que aumenta el porcentaje de los componentes químicos que forman los feldespatos $\left(\mathrm{Al}_{2} \mathrm{O}_{3}\right.$, $\mathrm{Na}_{2} \mathrm{O}, \mathrm{CaO}, \mathrm{MgO}, \mathrm{FeO}$ y $\mathrm{Fe}_{2} \mathrm{O}_{3}$ ).
Desde el punto de vista de la alteración, a medida que en estas rocas plutónicas aumenta el porcentaje de feldespatos, aumenta su proclividad al deterioro. Es decir, frente a las mismas condiciones ambientales, las 


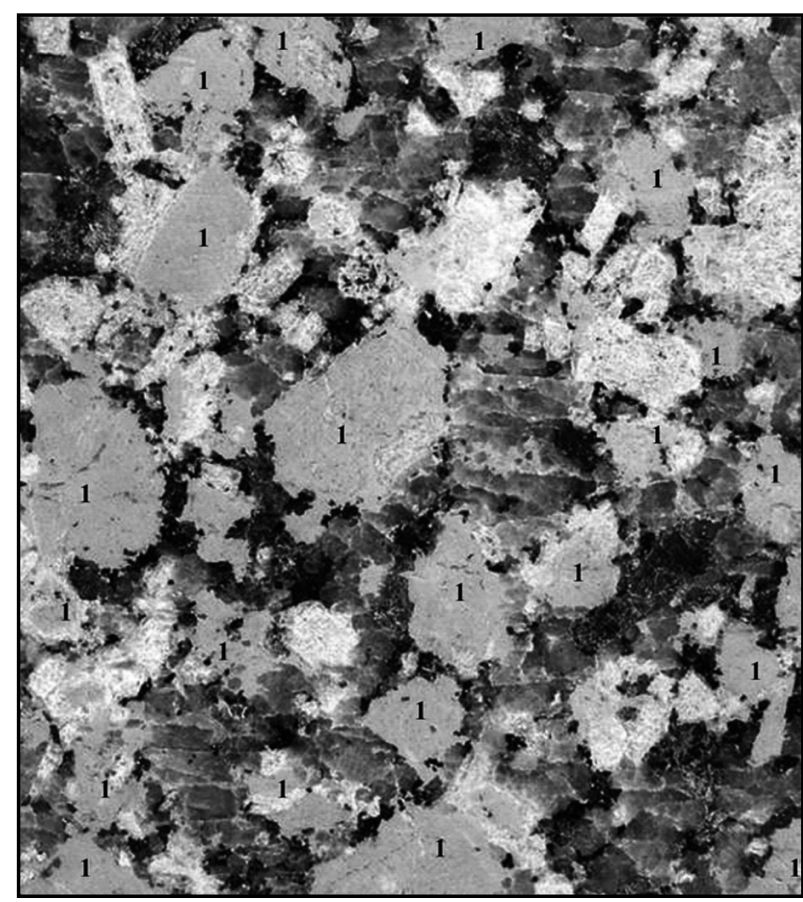

Figura 14. Aspecto macroscópico de un granito en el que los cristales de plagioclasa (1) se han teñido con cobaltinitrito sódico. Es un procedimiento sencillo y rápido para diferenciar la plagioclasa del resto de feldespatos.

granodioritas, sienitas y dioritas son más alterables que los granitos.

\subsection{Propiedades físicas del granito}

El granito, al igual que el resto de rocas utilizadas en edificación, se caracteriza por una serie de propiedades físicas que pueden medirse según diferentes procedimientos de ensayo normalizados. Entre estas propiedades citaremos como más importantes: la densidad, la porosidad $\mathrm{y}$, dentro de ella, la porosidad abierta $\left(\mathrm{n}_{0}\right)$, el contenido en agua por inmersión, la succión capilar, la permeabilidad al vapor de agua, la resistencia a la compresión uniaxial, flexión, resistencia al desgaste por rozamiento, resistencia al choque, etc. (Tabla 3) (7).

Algunas de estas propiedades físicas muestran distintos valores según su orientación en relación con las anisotropías texturales antes mencionadas (Tabla 4) (8-12), tal como ha podido constatarse en el granito de Axeitos (La Coruña).

Las propiedades físicas del granito varían según el grado de deterioro. En algunos elementos constructivos pueden realizarse tomogramas que aportan datos sobre alguna de las propiedades físicas del mismo como es el caso que se muestra en la Figura 15, obtenido mediante ultrasonidos. Corresponde a uno de los chantos del megalito de Axeitos (La Coruña) (13).

\section{FORMAS DE ALTERACIÓN DEL GRANITO}

Las formas de alteración más frecuentes que se suelen observar en el granito son: arenización, disgregación, escamas, placas, descamaciones, desplacaciones, alteración diferencial, eflorescencias salinas, fisuras, pátinas diversas: de tinción, de decoloración, biológicas (de líquenes, de musgo, etc.), depósitos superficiales, costras, costras biogénicas, abrasiones, etc. En su formación intervienen diferentes agentes de entre los cuales destaca el agua (líquida, sólida o en forma de vapor) como el más significativo. Las Figuras 16 a 20 ilustran las mencionadas patologías, cuya descripción se recoge en el glosario adjunto (14).

Tabla 2

Composición química de las principales rocas ígneas (Rangland, 1989)

\begin{tabular}{|c|c|c|c|c|c|}
\hline Plutónicas & Granito & Granodiorita & Sienita & Diorita & Gabro \\
\hline $\mathrm{SiO}_{2}$ & 71,3 & 66,1 & 58,6 & 57,5 & 50,1 \\
\hline $\mathrm{TiO}_{2}$ & 0,31 & 0,54 & 0,84 & 0,95 & 1,12 \\
\hline $\mathrm{Al}_{2} \mathrm{O}_{3}$ & 14,3 & 15,7 & 16,6 & 16,7 & 15,5 \\
\hline $\mathrm{Fe}_{2} \mathrm{O}_{3}$ & 1,21 & 1,38 & 3,04 & 2,50 & 3,01 \\
\hline $\mathrm{FeO}$ & 1,64 & 2,73 & 3,13 & 4,92 & 7,62 \\
\hline $\mathrm{MnO}$ & 0,05 & 0,08 & 0,13 & 0,12 & 0,12 \\
\hline $\mathrm{MgO}$ & 0,71 & 1,74 & 1,87 & 3,71 & 7,59 \\
\hline $\mathrm{CaO}$ & 1,84 & 3,83 & 3,53 & 6,58 & 9,58 \\
\hline $\mathrm{Na}_{2} \mathrm{O}$ & 3,68 & 3,75 & 5,24 & 3,54 & 2,39 \\
\hline $\mathrm{K}_{2} \mathrm{O}$ & 4,07 & 2,73 & 4,95 & 1,76 & 0,93 \\
\hline $\mathrm{P}_{2} \mathrm{O}_{5}$ & 0,12 & 0,18 & 0,29 & 0,29 & 0,24 \\
\hline
\end{tabular}




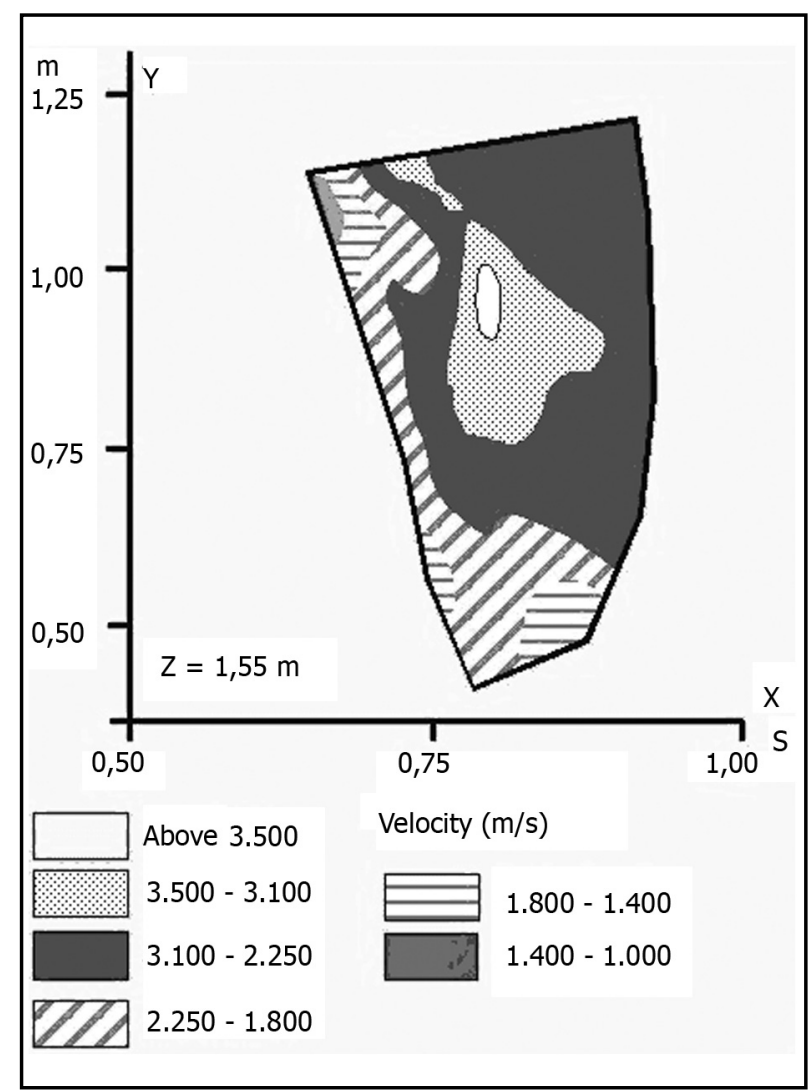

Figura 15. Tomografía ultrasónica realizada en un chanto del megalito de Axeitos (Coruña). El tomograma de la figura muestra la variación en la velocidad de propagación de ondas en relación con las anisotropías internas del elemento analizado.

\section{CAUSAS Y MECANISMOS DE DETERIORO DE LAS ROCAS GRANÍTICAS}

Varias son las causas y mecanismos por los cuales se produce el deterioro de las rocas graníticas a juzgar por las observaciones y análisis de las formas y productos de alteración, las características petrográficas y las propiedades físicas de estos materiales rocosos. En este sentido, se han agrupado las causas de la alteración y los mecanismos actuantes en tres grandes apartados, de acuerdo con los tipos de degradación generados en cada caso.

\subsection{Alteración física}

Es la alteración más importante de las que afectan al granito. La desagregación mecánica se produce a todos los niveles, y es observable tanto a escala microscópica (microdescamaciones, microfisuras, lajas) como a escala macroscópica (desplacaciones y macrofracturas). Los principales mecanismos generadores de este tipo de deterioro son las tensiones internas, inducidas o favorecidas en gran medida por la acción crioclástica de las sales solubles, los ciclos de calentamiento-enfriamiento, generados por la acción solar y también a veces, por los ciclos hielo-deshielo, cuando el agua, al descender la temperatura, es capaz de pasar a hielo (gelifracción).

El agua (humedad) es el vehículo imprescindible para el avance de la degradación física. La humedad es captada normalmente por la piedra en forma de vapor de agua o de agua líquida. Entre los posibles mecanismos de penetración de agua en las piedras, la condensación del vapor de agua es el más significativo. Otro mecanismo habitual es la ascensión capilar de agua del subsuelo y también la absorción de agua procedente de la lluvia y en muchos casos retenida en ciertas partes de los edificios (cornisas, alféizares de ventanas, solados de balcones...). En estos casos su presencia suele estar relacionada con un mal drenaje de los elementos constructivos.

a) Condensación superficial. Tiene lugar cuando la concentración de vapor de agua es la máxima posible para una temperatura dada. Cuando la temperatura

Tabla 3

Propiedades físicas de las rocas graníticas. Datos obtenidos de Roc Máquina (Esbert et al., 1997)

\begin{tabular}{|l|c|c|c|c|c|c|c|}
\hline & $\mathbf{\rho}_{\mathbf{d}}$ & $\mathbf{n}_{\mathbf{0}}$ & $\mathbf{W}$ & $\mathbf{R}_{\mathbf{C}}$ & $\mathbf{R}_{\mathbf{F}}$ & $\mathbf{R}_{\mathbf{D}}$ & $\mathbf{R}_{\mathbf{C H}}$ \\
\hline Rosa Porriño (Pontevedra) & 2,61 & $>0,8$ & 0,30 & 115 & 12 & 1,0 & 55 \\
\hline Rosa Mondariz (Pontevedra) & 2,64 & $>0,8$ & 0,30 & 110 & 15 & 1,0 & 55 \\
\hline Rosavel (Orense) & 2,63 & $>1,8$ & 0,70 & 63 & 18 & 0,04 & 45 \\
\hline Blanco Berrocal (Madrid) & 2,59 & $>0,5$ & 0,19 & 196 & 15 & 0,5 & 20 \\
\hline Crema Cabrera (Madrid) & 2,69 & $>0,8$ & 0,29 & 145 & 15 & 0,07 & 65 \\
\hline Blanco Cristal (Madrid) & 2,61 & $>0,7$ & 0,26 & 145 & 15 & 0,07 & 65 \\
\hline Gris Villa (Segovia) & 2,67 & $>0,5$ & 0,18 & 169 & 18 & 1,3 & 50 \\
\hline Gran Beige (Cáceres) & 2,63 & $>0,9$ & 0,34 & 132 & 10 & 0,2 & 60 \\
\hline Gris Quintana (Badajoz) & 2,66 & $>0,7$ & 0,25 & 138 & 10 & 1,6 & 50 \\
\hline Azul Arán (Lleida) & 2,55 & $>2,5$ & 1,00 & 91 & 10 & 0,5 & 135 \\
\hline
\end{tabular}

Datos recogidos de ROc Máquina. $\rho d$ : densidad de la roca seca $\left(\mathrm{g} / \mathrm{cm}^{3}\right), \mathrm{n} 0$ : porosidad abierta (\%), W: contenido en agua por inmersión (\%), RC: resistencia a la compresión uniaxial (MPa), RF: resistencia a la flexión (MPa), RD: resistencia al desgaste por rozamiento (mm), RCH: resistencia al choque (cm). 
Tabla 4

Propiedades físicas del granito de Axeitos (La Coruña) en relación con sus anisotropías texturales

\begin{tabular}{|c|c|c|}
\hline & Propiedades físicas & Valores medios \\
\hline Densidad $(\rho d)(8)$ & & $2585 \mathrm{~kg} / \mathrm{m}^{3}$ \\
\hline Porosidad abierta $\left(\mathrm{n}_{0}\right)(8)$ & & $2,0 \%$ \\
\hline Coeficiente de saturación en agua $\left(\mathrm{W}_{\mathrm{s}}\right)(8)$ & & $0,8 \%$ \\
\hline \multirow[t]{2}{*}{ Coeficiente de capliaridad (C) (9) } & Direccion $\mathrm{X}$ & $2,5 \times 10^{-4} \mathrm{~g} / \mathrm{cm}^{2} \cdot \mathrm{s}^{1 / 2}$ \\
\hline & Dirección Z & $2,9 \times 10^{-4} \mathrm{~g} / \mathrm{cm}^{2} \cdot \mathrm{s}^{1 / 2}$ \\
\hline \multirow[t]{2}{*}{ Coeficiente de permeabilidad al vapor de agua $\left(\mathrm{K}_{\mathrm{v}}\right)(10)$} & Dirección $\mathrm{X}$ & $0,25 \times 10^{-4} \mathrm{~g} / \mathrm{cm}^{2} \cdot 24 \mathrm{~h}$ \\
\hline & Dirección Z & $0,66 \times 10^{-4} \mathrm{~g} / \mathrm{cm}^{2} \cdot 24 \mathrm{~h}$ \\
\hline \multirow{2}{*}{ Hinchamiento lineal $\left(\varepsilon_{\mathrm{s}}\right)(11)$} & Dirección X & $0,004 \pm 0,0001 \%$ \\
\hline & Dirección Z & $0,034 \pm 0,004 \%$ \\
\hline \multirow[t]{2}{*}{ Resistenca a la compresión $\left(\sigma_{\mathrm{s}}\right)(12)$} & Dirección X & $101 \pm 23 \mathrm{MPa}$ \\
\hline & Dirección Z & $107 \pm 7,0 \mathrm{MPa}$ \\
\hline \multirow{2}{*}{ Resistencia a la tracción $\left(\sigma_{T}\right)(12)$} & Dirección X & $8,5 \pm 0,0 \mathrm{MPa}$ \\
\hline & Dirección Z & $9,0 \pm 0,5 \mathrm{MPa}$ \\
\hline
\end{tabular}

(8) RILEM 1980; (9) NORMAL 1981; (10) NORMAL 1985; (11) ISRM 1979; (12) SRM 1981.

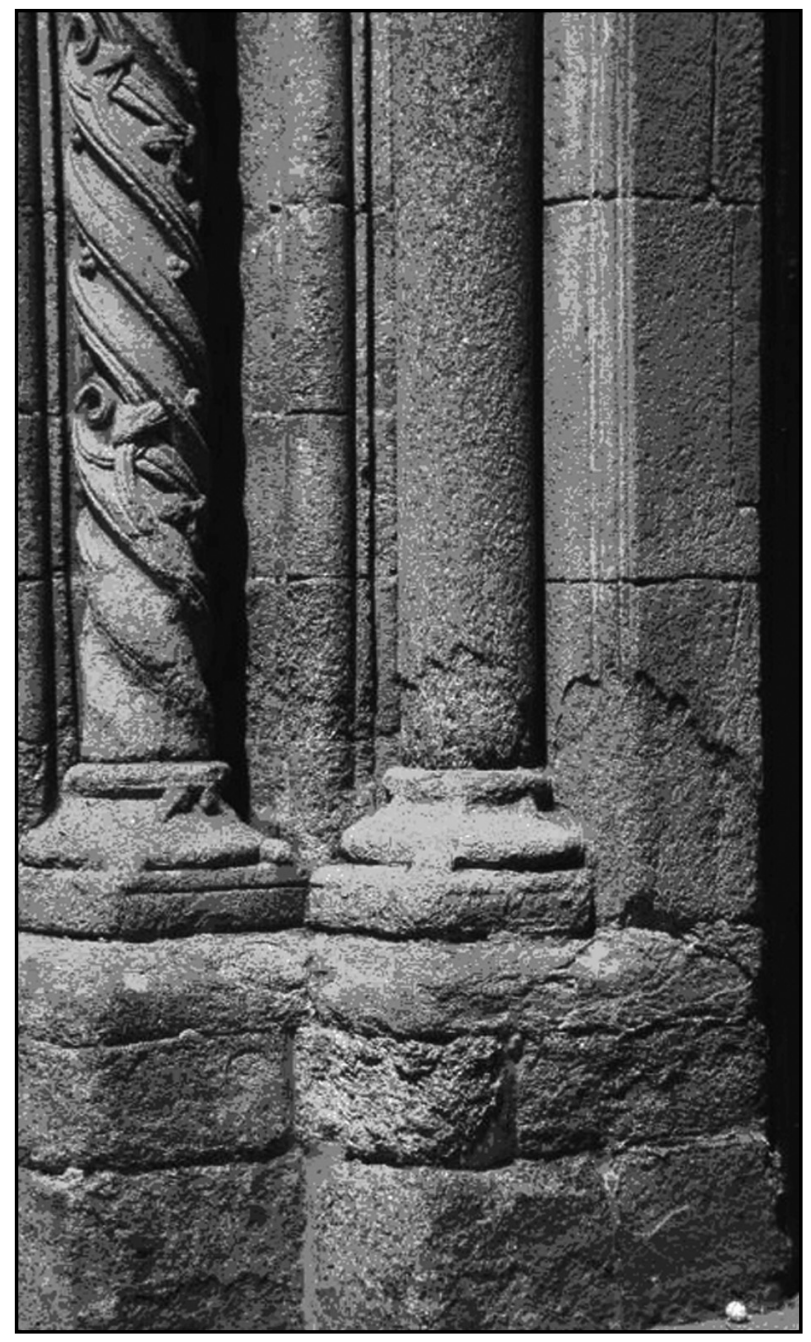

Figura 16. Abrasión y desplacaciones en el basamento y columnas situadas en la Puerta de Platerías de la Catedral de Santiago de Compostela.

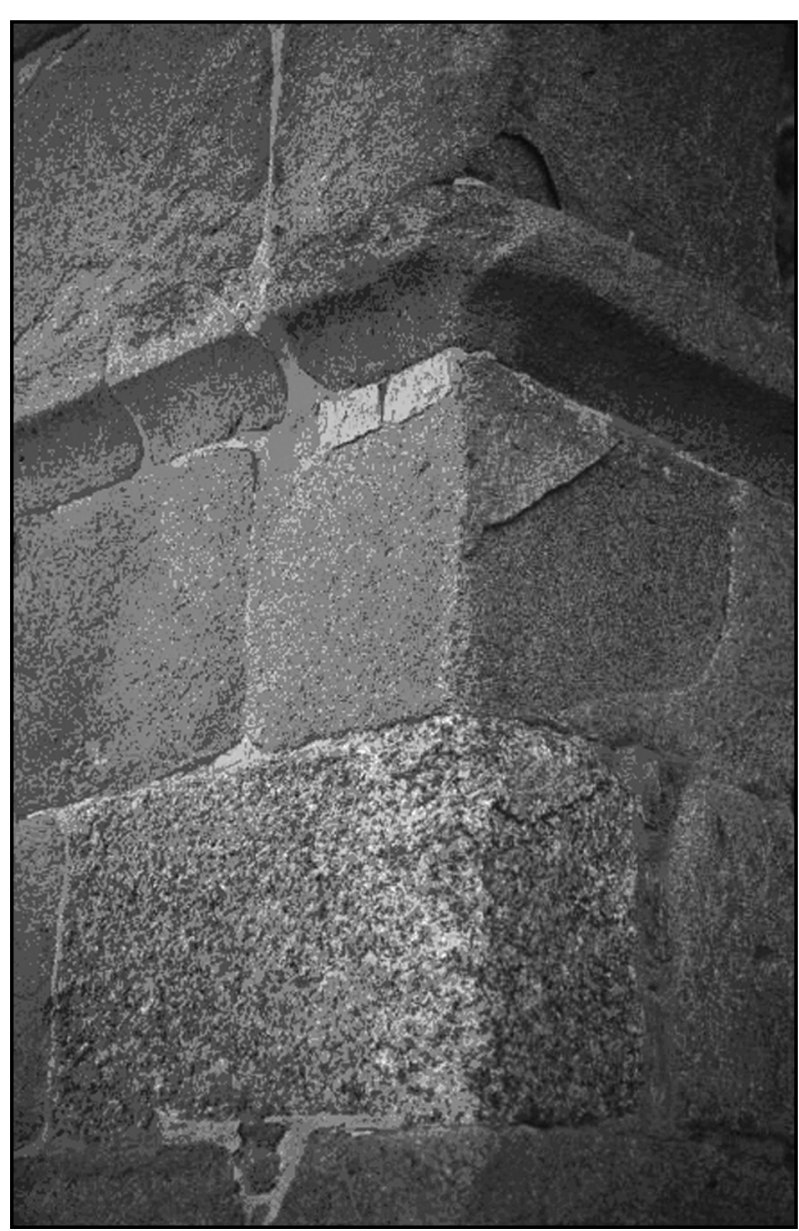

Figura 17. Alteración diferencial de dos bloques de granito, uno de grano fino con desplacaciones (parte superior) y otro de grano grueso con arenización (parte inferior). Catedral de Santiago de Compostela. 


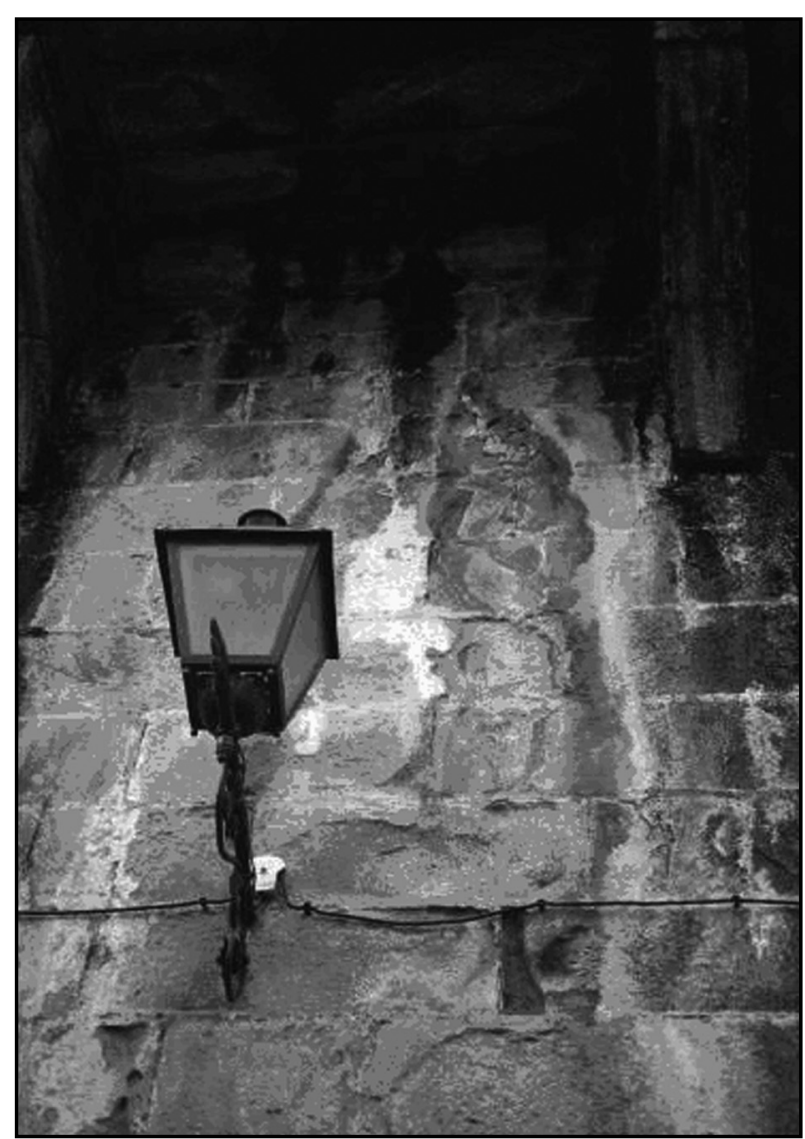

Figura 18. Costras negras situadas debajo de una terraza de la Catedral de Santiago. Plaza del Obradoiro.

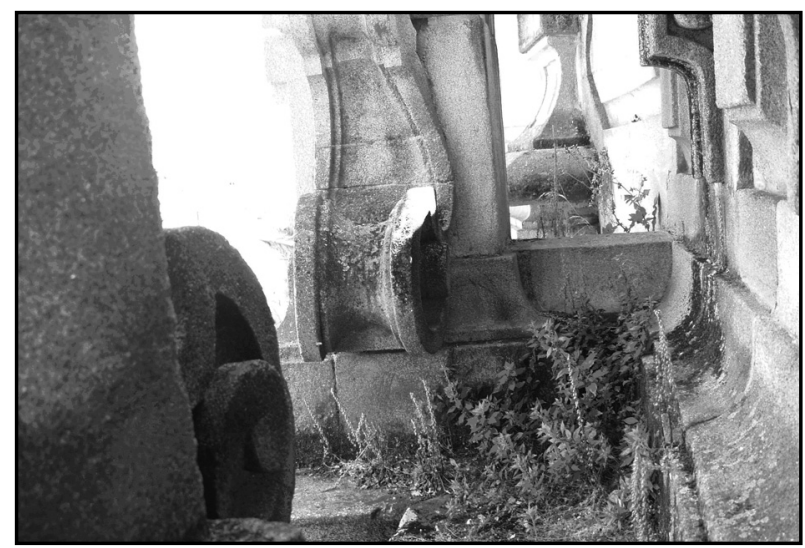

Figura 20. Especies vegetales implantadas en las cornisas de la Catedral de Ourense.

de la superficie de la piedra se halla por debajo del punto de rocío del aire circulante, el vapor de agua se condensa en su superficie. El agua líquida puede entonces moverse hacia el interior de la piedra aprovechando la red de microfisuras y poros. Hay que señalar que la abundante microfisuración preexistente observada en el granito, ligada a su historia geológica, favorece inicialmente la penetración del agua.

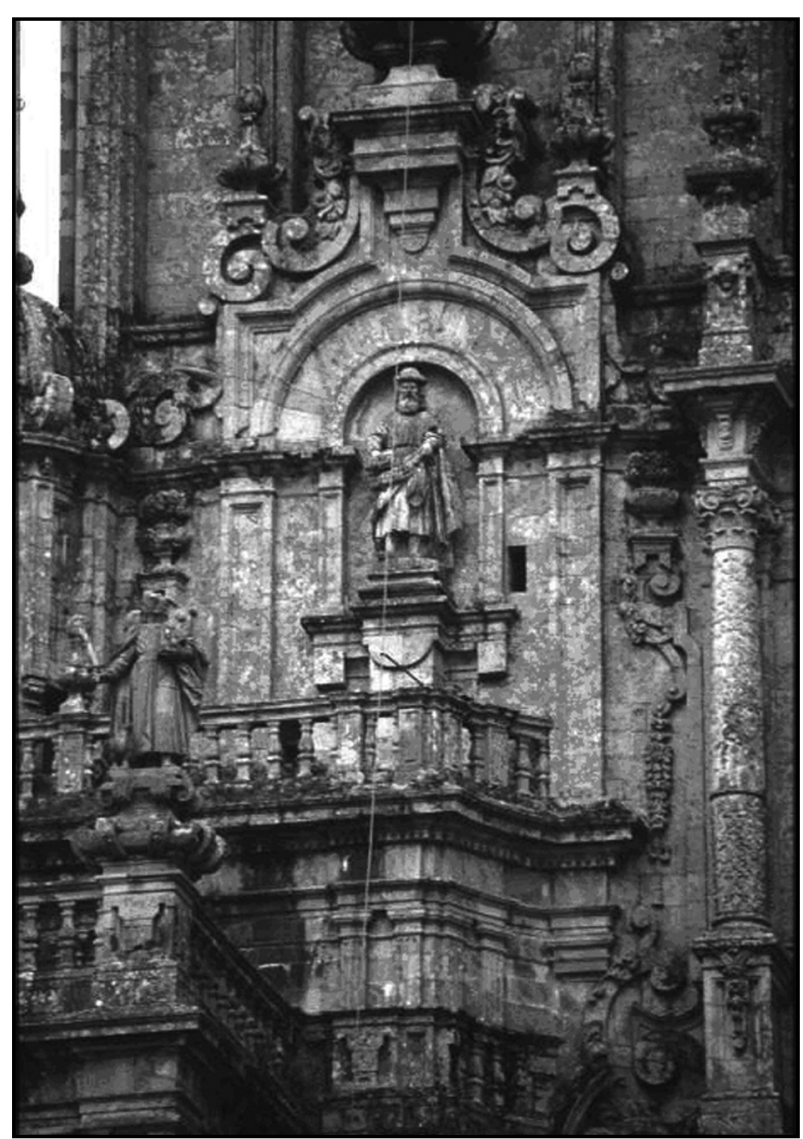

Figura 19. Pátina liquénica que coloniza casi toda la fachada principal de la Catedral de Santiago de Compostela.

Los fenómenos de condensación superficial son frecuentes en las paredes y muros exteriores de los edificios, especialmente en sus zonas bajas, afectando sobre todo a las dos o tres hileras de sillares inferiores. La condensación alcanza su máximo, por lo general, a últimas horas de la tarde y durante la noche, en las que las condiciones ambientales son las más propicias. A este respecto, la existencia de condiciones climáticas específicas alrededor del edificio (microclimas) parece ser determinante. La condensación superficial es estimulada por las humedades relativamente altas y las bajas temperaturas de superficie de las piedras. Esto último puede producirse por insuficiente insolación térmica de paredes y muros, bajas temperaturas en el exterior o excesiva ventilación. Todos estos supuestos se dan, en mayor o menor medida, dependiendo, en cada caso, de la situación y orientación particular de los elementos constructivos del edificio.

b) Condensación intersticial. Ocasionalmente la condensación de vapor de agua puede tener lugar dentro de la piedra si la humedad es muy alta y se alcanza el punto de rocío en algún punto del interior de la pared o muro. 
En cualquier casa, el agua condensada, superficial o intersticialmente, y retenida en el seno del material, puede eventualmente convertirse en hielo si la temperatura es lo suficientemente baja (heladas). En estas circunstancias es cuando se originan los mayores daños físicos en la piedra, los cuales conducen, a la larga, al progresivo debilitamiento y desagregación del material rocoso.

c) Agua de lluvia. El mecanismo de penetración de las aguas pluviales en la piedra es mucho más esporádico, en comparación con la condensación, y depende en gran medida de la construcción y condiciones de las superficies expuestas. Generalmente, la película de agua que se adhiere a la superficie pétrea es forzada a penetrar hacia el interior del muro por la presión del viento y la succión capilar. La penetración puede verse facilitada por la presencia en la piedra de fisuras, grietas y otras aberturas, las cuales actúan como vías de acceso de los fluidos externos.

\subsection{Alteración química}

El granito se halla sujeto a modificaciones o cambios composicionales por causa de la meteorización química natural. La descomposición mineral afecta principalmente a los feldespatos y concretamente a las plagioclasas, generando, como productos últimos de alteración, minerales arcillosos (p. ej. caolinita y halloysita). Estas transformaciones requieren un determinado grado de humedad, el cual es suministrado por los mecanismos anteriormente descritos.

Por otra parte, la acción de las sales solubles contribuye eficazmente al deterioro químicofísico del material rocoso. Dichas sales solubles pueden proceder de la interacción, en presencia de agua, de contaminantes atmosféricos con minerales de la roca, así como de la lixiviación y disolución parcial de los morteros de las juntas de los sillares por las aguas aciduladas circulantes.

Respecto a los contaminantes atmosféricos parece ser el $\mathrm{SO}_{2}$ y los cloruros los que contribuyen prioritariamente al deterioro, con la formación de costras de naturaleza sulfatada (yeso) en el primer caso y con la disgregación de los materiales graníticos en el segundo.

\subsection{Alteración biológica}

La implantación de organismos vivos en la superficie de las piedras o cerca de ellas (especialmente en hendiduras y en la parte posterior de escamas y placas) puede tener su influencia en la degradación física y química de las piedras graníticas, especialmente en regiones húmedas.

En las partes de los edificios, construidas con granito, en las que el drenaje de agua es defectuoso (solados, cornisas, paramentos horizontales, etc.), suelen detectarse verdaderas colonizaciones biológicas. El casco urbano de Santiago de Compostela es un ejemplo característico.

Los organismos vivos asentados sobre el granito crean zonas con retenciones diferenciales de humedad. Su actividad metabólica contribuye también al deterioro diferencial de los minerales que forman el granito.

Más detalles sobre la alteración biológica se pueden encontrar en: Caneva et al., 1991 (15).

\section{AGRADECIMIENTOS}

Al Consorcio de Santiago por su invitación a participar como ponente en el II Congreso Internacional de Arquitectura que con el título "Antiguos espacios para nuevos tiempos. El material pétreo y sus fábricas en el patrimonio" se celebró en Santiago de Compostela del 25 al 27 de noviembre de 2004 . El trabajo presentado en dicha ponencia fue el germen para la elaboración de este artículo.

A los profesores del Área de Petrología y Geoquímica de la Universidad de Oviedo, Dres. Modesto Montoto, Fco Javier Alonso, Luis Miguel Suárez del Rió y a D. a Araceli Rojo Álvarez, geóloga de la empresa GEA, por haberme facilitado algunas de las fotografías que aparecen en el texto.

También al profesor Jorge Ordaz, de la anteriormente citada Área de Petrología y Geoquímica, coautor del Glosario de Términos que se incluye al final de este trabajo.

A la CICYT por la financiación de diferentes proyectos de investigación, en especial el último: "MAT-2004-06804C02-01".

\section{BIBLIOGRAFÍA}

(1) EN 12670:2001: Piedra natural: terminología.

(2) Consejo Superior de Investigaciones Científicas: Actas del workshop: alteración de granitos y rocas afines, empleados como materiales de construcción, 1993, 190 pp.

(3) Chabas, A. y Jeannette, D.: "Weathering of marbles and granites in marine environment: petrophysical properties and special role of atmospheric salts", Environmental Geology, vol. 30, nº 3 (2001), pp. 359-368. 
(4) Bates, R. L.: Geology of the industrial rocks and minerals, Dover Publications Inc. New York, 1969, 459 pp.

(5) Esbert, R. M.; Pérez Ortiz, A; Ordaz, J. y Alonso, F. J.: "Intrinsic factors influencing the decay of the granite as a building stone", VII th Intl. Congres. Int. Associations of Engineering Geology (1994), pp. 3659-3665.

(6) Ragland, P. C.: "Basic Analytical Petrology", Oxford University Press, 1989, 369 pp. Silva, Benita.

(7) Esbert, R. M.; Ordaz, J.; Alonso, F. J., Montoto, M.; Gonzales, T. y Álvarez del Buergo, M.: "Manual de diagnosis y tratamiento de materiales pétreos y cerámicos", Col.legi d'Aparelladors i Arquitectes Tècnics de Barcelona, 1997, 126 pp.

(8) RILEM 1980: "Essais recommandés pour mesurer l'altération des pierres et evaluation l'eficacité des methods de traitment/Recommended test to measure the deterioration of stone and to assess the effectiveness of treatment methods", Matériaux et Constructions, Bull. RILEM, 13(75), pp. 216-220.

(9) NORMAL 1981: Assorbimento d'acqua per immersione totale e capacitá d'imbibizione, CNR-IRC, Roma, 5 pp.

(10) NORMAL 1985: Permeabilitá al vapour d'acqua, CNR-ICR, Roma, 6 pp.

(11) ISRM 1979: "Suggested methods for the quantitative description of discontinuities in rock masses", Int. J. Rock Mech. Min. Sci. \& Geomech. Abstr., vol. 15, Great Britain, pp. 319-368.

(12) ISRM 1981: Rock characterization testing and monitoring ISRM suggested methods. E.T. Brown, Ed., Pergamon Press, Londres, $211 \mathrm{pp}$.

(13) Valdeón, L.; Montoto, M.; Calleja, L. y Esbert, R. M.: "A method to assess spatial coordinates in art and archaeological objects: Application of tomography to a dolmen", J. Archaeological Sciences, no 24 (4) (1997), pp. 337-346.

(14) Ordaz y Esbert: "Glosario de términos relacionados con el deterioro de las piedras de construcción", Mater. Construcc., vol. 38, no 209 (1988), pp. 39-45.

(15) Caneva, G.; Nugari, M. P. y Salvadori, O.: "Biology in the conservation of Works of Art", ICCROM, 1991, 182 pp.

$* * *$

\section{Glosario de términos relacionados con el deterioro de las piedras de construcción}

Abrasión: desgaste de la superficie de los materiales rocosos, por fricción o impacto, originado por la acción de los agentes erosivos (viento, lluvia, etc.).

Arenización: tipo de meteorización caracterizado por la caída "grano a grano", espontánea o inducida, de material tamaño arena.

Alteración diferencial: modificación o cambio en la composición química o mineralógica de un material pétreo, producido por los agentes meteóricos.

Costra: lámina compacta de material en la parte externa de una piedra, producto de una transformación superficial, y cuya naturaleza químico-mineralógica y características físicas son parcial o totalmente distintas de las del substrato pétreo sobre el que se asienta. Visualmente se distinguen, en general, por sus rasgos morfológicos (a veces en forma de caparazón), dureza (se hallan endurecidas con respecto al material rocoso alterado); y, a menudo, por su color (costras negras), al contener productos carbonosos de contaminación (hollín, polvo, etc.).

Costra biogénica: tipo de costra en la que abunda la presencia de vegetación (líquenes, musgo, etc.).

Depósito superficial: acumulación de material de origen diverso (polvo, humos, hollín, guano, microorganismos, etc.) en la superficie de una piedra. Normalmente de escasa cohesión, espesor variable y baja adherencia al substrato sobre el que se asienta.

Descamación: levantamiento y separación de escamas paralelamente a la superficie de la piedra, por causa de diferentes mecanismos (cambios de temperatura o humedad, acción del hielo o de las sales, etc.).

Desplacación: levantamiento y separación de placas.

Eflorescencia salina: capa o formación de cristales de sales solubles, de color blanquecino, no muy consistente, que se forma en la superficie de una piedra porosa, debida a fenómenos de migración y evaporación de agua conteniendo sales solubles. 
Escama: lámina o plaquita más o menos compacta, de unos pocos milímetros de espesor, que se desprende paralelamente a la superficie de la piedra, e independientemente de la estructura de la misma.

Fisura: discontinuidad planar, macroscópica o microscópica (microfisura), de diverso origen y dimensiones variables. En general, fractura o hendidura en la masa de una piedra. Se pueden distinguir varios tipos: las fisuras preexistentes, originales de la roca ("pelos" en cantería); las producidas durante la extracción, labra, esculpido, aserrado o manipulación en general de una piedra ("artefactos"); las generadas a consecuencia de esfuerzos mecánicos derivados de la estructura del edificio (p. ej. asentamiento defectuoso de un sillar, anclajes internos, etc.); y las inducidas por los ciclos térmicos, de hielo-deshielo, de humedad-sequedad, etc.

Pátina: capa o película superficial y delgada que se forma sobre las piedras por diversas causas. Modificación superficial del material que no implica necesariamente procesos de degradación o deterioro. El término genérico de "pátina" incluye varias acepciones:

a) Tonalidad o aspecto externo que cualquier piedra adquiere por "envejecimiento" con el paso del tiempo, y bajo los efectos de la intemperie.

b) Decoloración debida a causas naturales o artificiales.

c) Película coloreada artificial (patinación).

d) Película de carácter orgánico (p. ej. algas) de tonalidad variable (pátina biológica).

e) Teñido superficial debido a diversas substancias: orín, verdín, etc. (pátina de tinción).

f) Acumulación superficial de suciedad (pátina de ennegrecimiento).

Placa: lámina compacta más o menos alargada de varios milímetros de espesor. 